Erratum

\title{
Erratum to "NADPH Oxidase-Induced NALP3 Inflammasome Activation Is Driven by Thioredoxin-Interacting Protein Which Contributes to Podocyte Injury in Hyperglycemia"
}

\author{
Pan Gao, ${ }^{1}$ Fang-Fang He, ${ }^{1}$ Hui Tang, ${ }^{1}$ Chun-Tao Lei, ${ }^{1}$ Shan Chen, ${ }^{1}$ Xian-Fang Meng, ${ }^{2}$ \\ Hua Su, ${ }^{1}$ and Chun Zhang ${ }^{1}$ \\ ${ }^{1}$ Department of Nephrology, Union Hospital, Tongji Medical College, Huazhong University of Science and Technology, \\ Wuhan 430022, China \\ ${ }^{2}$ Department of Neurobiology, Tongji Medical College, Huazhong University of Science and Technology, Wuhan 430030, China \\ Correspondence should be addressed to Hua Su; suhua7707@163.com and Chun Zhang; drzhangchun@hust.edu.cn \\ Received 12 December 2015; Accepted 5 January 2016
}

Copyright (c) 2016 Pan Gao et al. This is an open access article distributed under the Creative Commons Attribution License, which permits unrestricted use, distribution, and reproduction in any medium, provided the original work is properly cited.

In Figure 6 in the paper titled "NADPH Oxidase-Induced NALP3 Inflammasome Activation Is Driven by ThioredoxinInteracting Protein Which Contributes to Podocyte Injury in Hyperglycemia" [1], Figures 6(a) and 6(c) are completely the same pictures. This correction does not have any influence on the results and discussions of the study, which are correctly described in the text. In the published version of Gao et al., 2015, Figure 6(a) should be the representative Western blot graph of the expression of gp91 ${ }^{\text {phox }}$ in HG-exposed podocytes without or with TXNIP shRNA transfection. Below is the correct version of Figure 6.

\section{References}

[1] P. Gao, F.-F. He, H. Tang et al., "NADPH oxidase-induced NALP3 inflammasome activation is driven by thioredoxininteracting protein which contributes to podocyte injury in hyperglycemia," Journal of Diabetes Research, vol. 2015, Article ID 504761, 12 pages, 2015. 


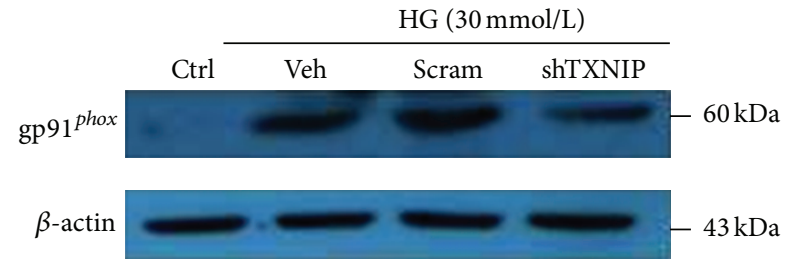

(a)

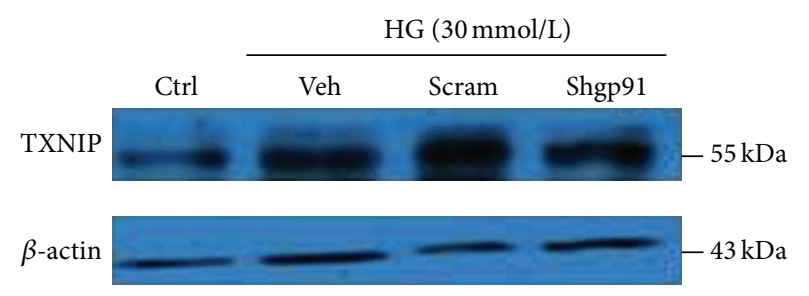

(c)

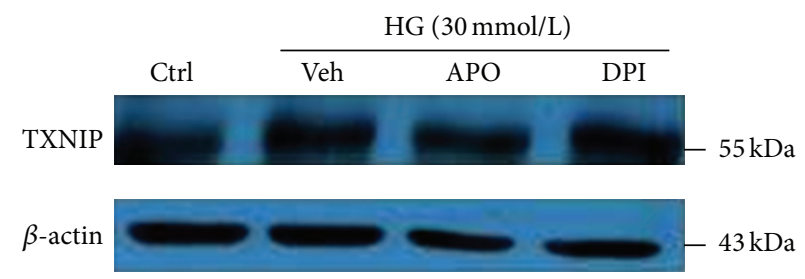

(e)

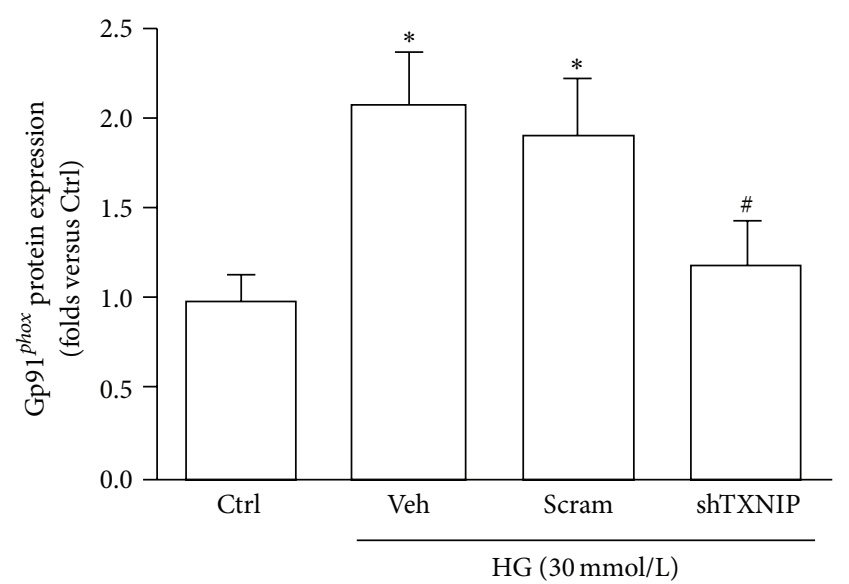

(b)

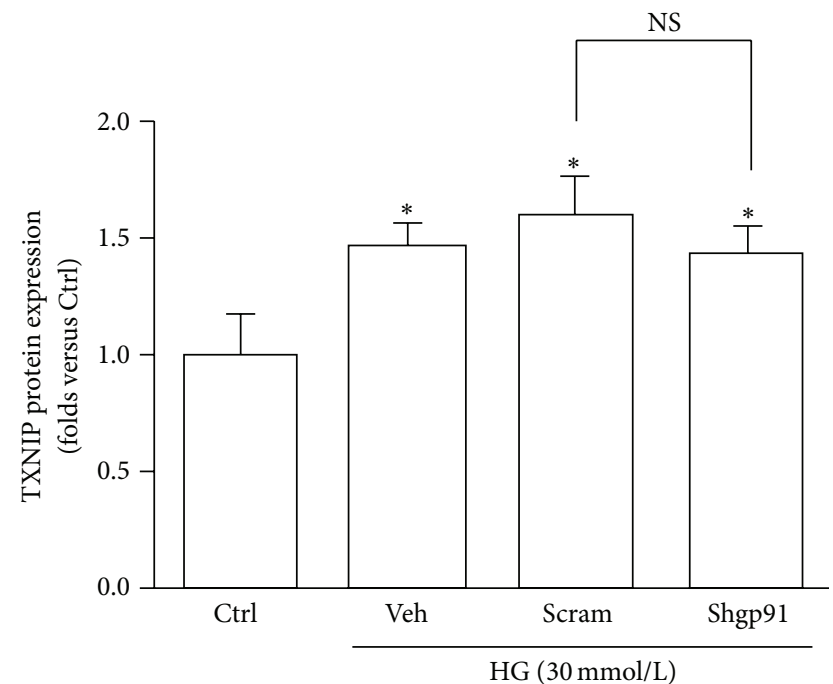

(d)

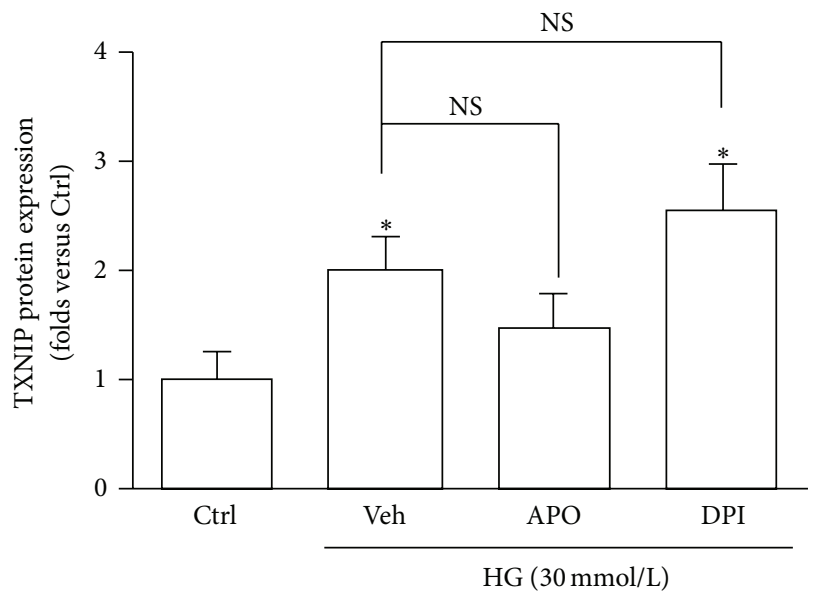

(f)

FIGURE 6: Inhibition of TXNIP abolishes the HG-triggered upregulation of gp91 ${ }^{\text {phox }}$. (a) Western blot analysis showing the expression of gp91 ${ }^{\text {phox }}$ in HG-exposed podocytes without or with TXNIP shRNA transfection. (b) Summarized data showing the band intensities measured from gp91 phox $(n=6)$. (c) Western blot analysis showing the expression of TXNIP in HG-stimulated podocytes without or with gp91 ${ }^{\text {phox }}$ shRNA transfection. (d) Summarized data showing the band intensities measured from TXNIP $(n=6)$. (e) Protein expression of TXNIP in HG-stimulated podocytes without or with pretreatment of APO or DPI. (f) Summarized data showing the band intensities of TXNIP $(n=4-6)$. Ctrl: control; HG: high glucose; Veh: vehicle; Scram: scrambled shRNA; shTXNIP: TXNIP shRNA; shgp91: gp91 ${ }^{\text {phox }}$ shRNA; APO: apocynin; DPI: diphenyleneiodonium. ${ }^{*} P<0.05$ compared with $\mathrm{Ctrl} ;{ }^{\#} P<0.05$ compared with HG group treated with vehicle or transfected with scramble shRNA. 


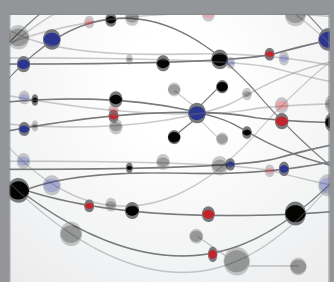

The Scientific World Journal
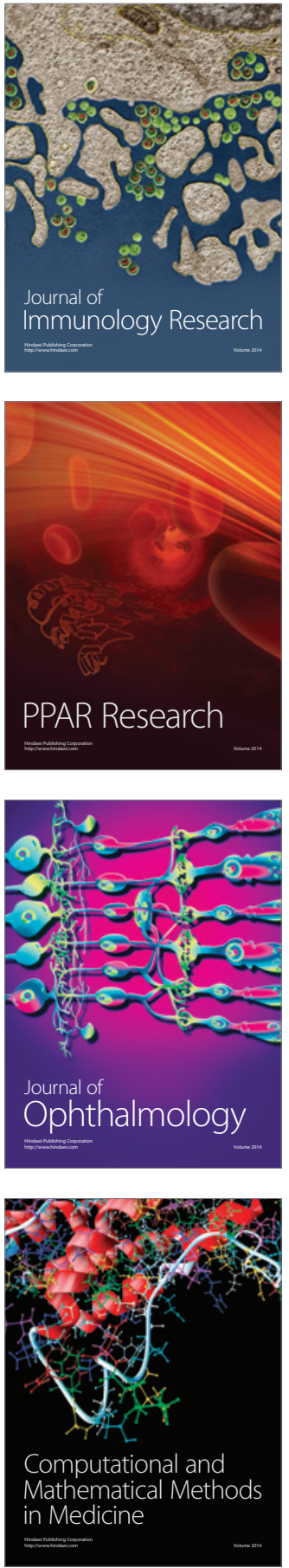

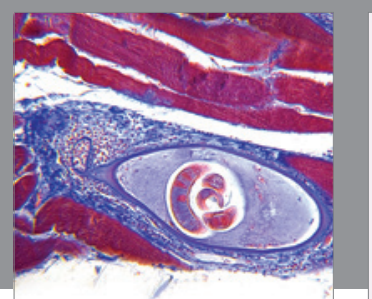

Gastroenterology Research and Practice

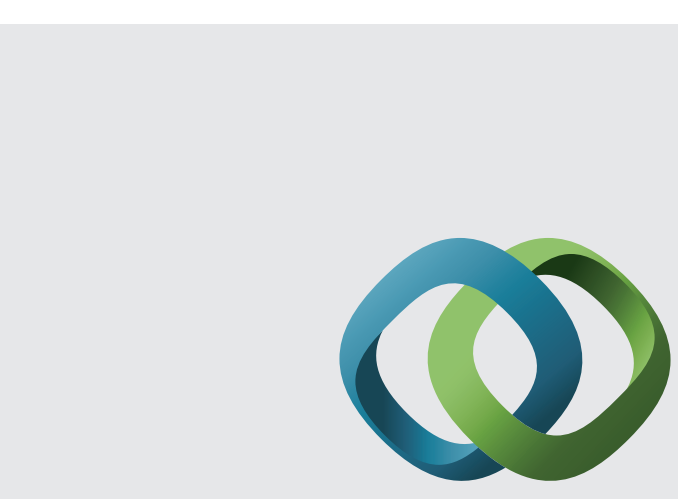

\section{Hindawi}

Submit your manuscripts at

http://www.hindawi.com
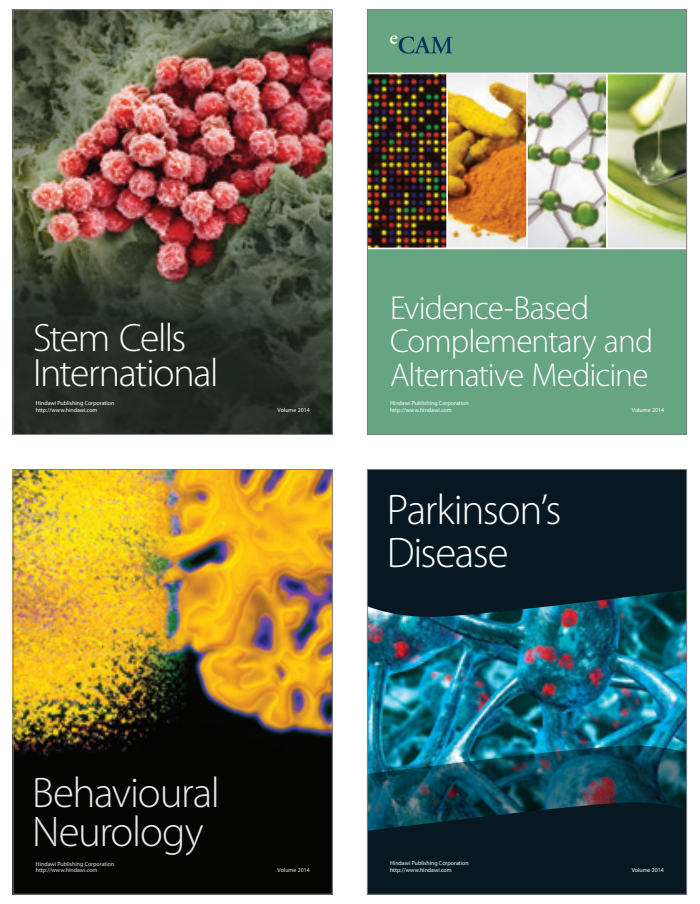
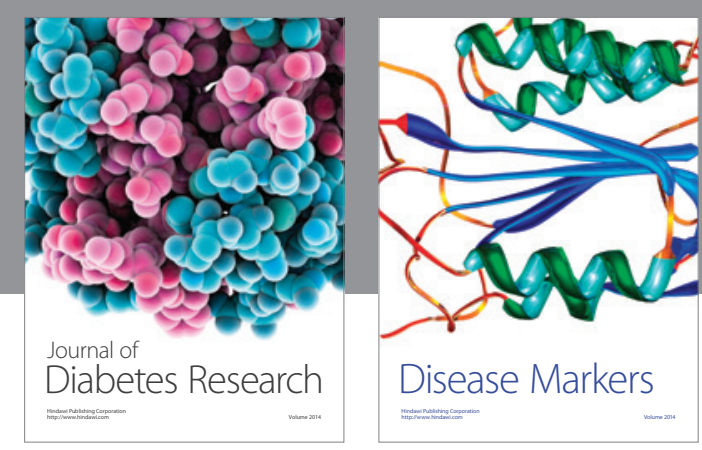

Disease Markers
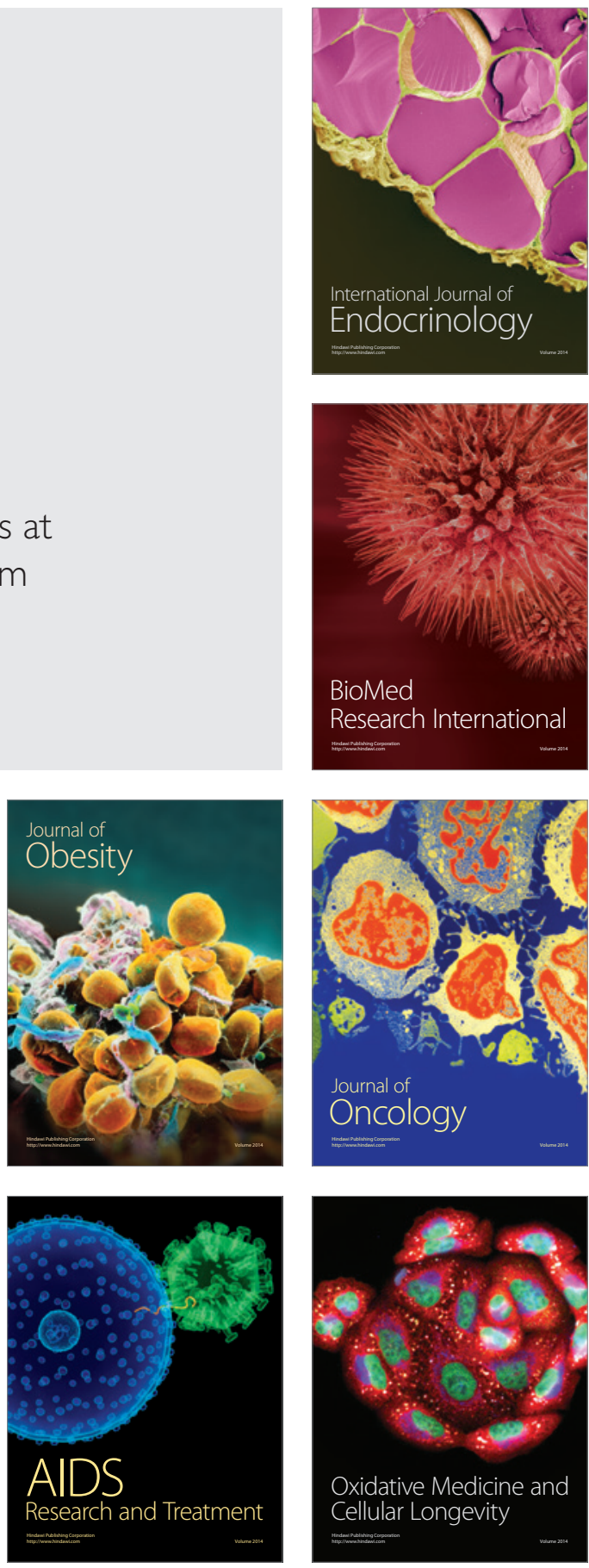